PROCEEDINGS OF THE

AMERICAN MATHEMATICAL SOCIETY

Volume 140, Number 9, September 2012, Pages 3237-3245

S 0002-9939(2012)11159-1

Article electronically published on January 26, 2012

\title{
SPECTRAL PERMANENCE FOR THE MOORE-PENROSE INVERSE
}

\author{
DRAGAN S. DJORDJEVIĆ, SNEŽANA Č. ŽIVKOVIĆ-ZLATANOVIĆ, \\ AND ROBIN E. HARTE \\ (Communicated by Marius Junge)
}

\begin{abstract}
C* algebra "spectral permanence" extends from the ordinary inverse to the Moore-Penrose inverse.
\end{abstract}

\section{INTRODUCTION}

"Spectral permanence" for $\mathrm{C}^{*}$ algebras says that if $T: A \rightarrow B$ is an isometric $\mathrm{C}^{*}$ homomorphism, then the image spectrum $\sigma_{B}(T a)$ is always the same as the original spectrum $\sigma_{A}(a)$ : equivalently, invertibility $T a \in B^{-1}$ implies invertibility $a \in A^{-1}$. In this paper we extend this to relative regularity and offer a fresh proof of the Harte-Mbekhta theorem ([5, Theorem 6]) which says that relatively regular $\mathrm{C}^{*}$ algebra elements always have "Moore-Penrose inverse". Instead of [5], the "poor man's path" between projections, we proceed via the Drazin inverse.

\section{Spectral Permanence}

Suppose $T: A \rightarrow B$ is a homomorphism of semigroups. Here "semigroup" $A$ is assumed to have an identity 1 . We write $A^{-1}$ for its subgroup of invertibles. Then there is the inclusion

$$
T\left(A^{-1}\right) \subseteq B^{-1} \subseteq B
$$

and hence also

$$
A^{-1} \subseteq T^{-1}\left(B^{-1}\right) \subseteq A .
$$

Now if there is equality in (1.2), we shall say that $T$ has the Gelfand property,

$$
A^{-1}=T^{-1}\left(B^{-1}\right) \text {. }
$$

This famously is the case when $A$ is a commutative Banach algebra and $T: A \rightarrow$ $B=C(X)$ is the Gelfand mapping from $A$ to the continuous functions on the "maximal ideal space" $X=\sigma(A)$ of $A$. Another familiar example is the left regular representation

$$
T=L: a \mapsto L_{a} \quad\left(A \rightarrow A^{A} \equiv \operatorname{Map}(A, A)\right),
$$

where, for each $x \in A$,

$$
L_{a}(x)=a x
$$

Received by the editors July 9, 2010 and, in revised form, March 30, 2011.

2000 Mathematics Subject Classification. Primary 46H05, 47A05, 47A53.

Key words and phrases. Drazin inverse, Moore-Penrose inverse, Fredholm theory, $\mathrm{C}^{*}$ algebras.

The authors are supported by the Ministry of Science of Serbia, grant \#174007.

(C)2012 American Mathematical Society 
This holds also for the natural embedding

$$
A=\operatorname{comm}_{B}(K) \subseteq B
$$

of the commutant of a subset $K \subseteq B$,

$$
\operatorname{comm}_{B}(K)=\{b \in B: a \in K \Longrightarrow b a=a b\} .
$$

The same is also true, among rings, of the quotient mapping

$$
T: A \rightarrow A / \operatorname{Rad}(A) \text {, }
$$

where

$$
\operatorname{Rad}(A)=\left\{a \in A: 1-A a \subseteq A^{-1}\right\}
$$

is the Jacobson radical of the ring $A$. Our final example, if not the most "elementary", is the process of taking the determinant of a square matrix: it is precisely because of the Gelfand property that it "determines" whether or not a matrix is invertible.

Generally when $A$ is a complex linear algebra there is a spectrum

$$
\sigma_{A}(a)=\left\{\lambda \in \mathbf{C}: a-\lambda \notin A^{-1}\right\}
$$

for elements $a \in A$; thus complex analysis is harnessed to the theory of invertibility. Now inclusion (1.1) takes the form

$$
\sigma_{B}(T a) \subseteq \sigma_{A}(a)
$$

while equality (1.3) corresponds to equality in (1.10), where it is referred to as "spectral permanence". Generally if a homomorphism $T: A \rightarrow B$ of Banach algebras is isometric, or more generally bounded below, then there is the inclusion

$$
\partial \sigma_{A}(a) \subseteq \sigma_{B}(T a) \subseteq \sigma_{A}(a),
$$

where we write $\partial K$ for the topological boundary of $K \subseteq \mathbf{C}$ : thus the condition, for a particular $a \in A$, that

$$
\sigma_{A}(a) \subseteq \partial \sigma_{A}(a)
$$

is sufficient for "spectral permanence at $a$ " in the sense of equality in (1.10).

\section{Generalized Permanence}

If $A$ is a semigroup, we shall write

$$
A^{\cap}=\{a \in A: a \in a A a\}
$$

for the "regular" or relatively regular elements of $A$, those $a \in A$ which have a generalized inverse $b \in A$ for which

$$
a=a b a .
$$

We remark that if $(2.2)$ holds, the products

$$
p=b a=p^{2}, q=a b=q^{2}
$$

are both idempotent. Generally if $T: A \rightarrow B$ is a homomorphism, there is the inclusion

$$
T\left(A^{\cap}\right) \subseteq B^{\cap} \subseteq B
$$

and hence also

$$
A^{\cap} \subseteq T^{-1}\left(B^{\cap}\right) \subseteq A .
$$


If there is equality in (2.4), we shall say that $T$ has generalized permanence. This happens for example when

$$
T^{-1}(0) \subseteq A^{\cap}, T(A)=B .
$$

Recall the implication

$$
(a-a A a) \cap A^{\cap} \neq \emptyset \Longrightarrow a \in A^{\cap} .
$$

If in particular there is $b \in A$ for which

$$
a-a b a=0=a b-b a,
$$

then $a \in A$ is very special; we shall say that $a \in A$ is simply polar and refer to the product $b a b$ as the group inverse for $a \in A$. More generally a group inverse for a power $a^{n}$ gives rise to a Drazin inverse for $a$. We remark that for $a \in A$ to be simply polar it is necessary and sufficient that

$$
a \in a^{2} A \cap A a^{2} .
$$

Indeed [8] there is the implication

$$
a^{2} u=a=v a^{2} \Longrightarrow a u=v a, a u a=a=a v a,
$$

giving (2.6) with $b=v a u$. In particular if $A=B(X)$ for a Banach space $X$, then relatively regular elements have closed range.

Theorem 2.1. If $a \in A=B(X)$ for a normed space $X$, then

$$
a \in A^{\cap} \Longrightarrow a(X)=\operatorname{cl} a(X) \text {. }
$$

If $X$ is complete, then for $a \in A$ to be simply polar it is necessary and sufficient that it has ascent $\leq 1$,

$$
\left.a^{-2}(0) \subseteq a^{-1}(0) \text { (equivalently } a^{-1}(0) \cap a(X)=O \equiv\{0\}\right)
$$

and also descent $\leq 1$,

$$
\left.a(X) \subseteq a^{2}(X) \text { (equivalently } a^{-1}(0)+a(X)=X\right) .
$$

Proof. If $a=a b a \in A$, then the range

$$
a(X)=a b(X)=(1-a b)^{-1}(0)
$$

is the null space of the complementary idempotent, therefore closed. Now the complementary subspaces $a^{-1}(0)$ and $a(X)$ determine the idempotent $q: X \rightarrow X$, defined by setting

$$
q(x) \in a(X), \quad x-q(x) \in a^{-1}(0)
$$

for each $x \in X$, whose boundedness, together with the closedness of the range $a(X)$, follows ([4, Theorem 4.8.2]) from the open mapping theorem, and finally, if $x \in X$,

$$
b(x)=b q(x), \quad b(a x)=q(x) .
$$

We remark, even together with the assumption $a \in A^{\cap}$, that the conditions (2.10) and $(2.11)$ are (4, 7.3.6.8]) not sufficient for simple polarity (2.6) when $A=B(X)$ for an incomplete normed space $X$.

More generally, if there is $n \in \mathbf{N}$ for which $a^{n}$ is simply polar, we shall also say that $a \in A$ is "polar" or Drazin invertible. If $a \in A$ is polar, then there is $b \in A$ for which $a b=b a$ and $a-a b a$ is nilpotent. 


\section{DRAZIN PERMANENCE}

More generally if we write

$$
\mathrm{QN}(A)=\left\{a \in A: 1-\mathbf{C} a \subseteq A^{-1}\right\}
$$

for the quasinilpotents of $A$, then $a \in \mathrm{QN}(A)$ if and only if

$$
\sigma_{A}(a) \subseteq\{0\},
$$

while with some complex analysis we can prove that if $a \in \mathrm{QN}(A)$, then

$$
\left\|a^{n}\right\|^{1 / n} \rightarrow 0 \quad(n \rightarrow \infty) .
$$

In the ultimate generalization of "group invertibility", we shall write $\mathrm{QP}(A)$ for the quasipolar elements $a \in A$, those which have a spectral projection $p \in A$ for which

$$
p=p^{2}, \quad a p=p a, \quad a+p \in A^{-1}, \quad a p \in \mathrm{QN}(A) .
$$

Now [7] the spectral projection and the Koliha-Drazin inverse

$$
a^{\bullet}=p, a^{\times}=(a+p)^{-1}(1-p)
$$

are uniquely determined and lie in the double commutant of $a \in A$. It is easy to see that if (3.3) is satisfied, then

$$
0 \notin \operatorname{acc} \sigma_{A}(a) ;
$$

the origin cannot be an accumulation point of the spectrum. Conversely if (3.5) holds, then we can display the spectral projection as a sort of "vector-valued winding number"

$$
a^{\bullet}=\frac{1}{2 \pi i} \oint_{\gamma(0)}(z-a)^{-1} d z
$$

where we integrate counterclockwise around a small circle $\gamma=\gamma(0)$, centre the origin, whose connected hull is a disc $\eta \gamma$ whose intersection with the spectrum is at most the point $\{0\}$. By the same technique we can display the Koliha-Drazin inverse in the form

$$
a^{\times}=\frac{1}{2 \pi i} \oint_{\gamma(\sigma(a) \backslash\{0\})} z^{-1}(z-a)^{-1} d z .
$$

Now generally for a homomorphism $T: A \rightarrow B$ there is the inclusion

$$
T \mathrm{QP}(A) \subseteq \mathrm{QP}(B),
$$

while if $T: A \rightarrow B$ has spectral permanence in the sense of (1.3), then it is clear from (3.5) that there is also "Drazin permanence" in the sense that

$$
\mathrm{QP}(A)=T^{-1} \mathrm{QP}(B) \subseteq A .
$$

Theorem 3.1. For Banach algebra homomomorphisms $T: A \rightarrow B$ there is the implication

$$
\text { spectral permanence } \Longrightarrow \text { Drazin permanence. }
$$

Proof. Equality in (1.10) together with (3.5) proves this.

In general we cannot deduce "generalized permanence", equality in (2.4), from the spectral permanence of (1.3): 
Theorem 3.2. If $T: A \rightarrow B$ and $A$ is commutative, then there is the inclusion

$$
T\left(A^{\cap}\right) \subseteq \mathrm{QP}(B) \cap B^{\cap} .
$$

Thus if $A$ is commutative, there is the implication, for arbitrary $a \in A$,

$$
T(a) \in B^{\cap} \backslash \mathrm{QP}(B) \Longrightarrow a \notin A^{\cap} .
$$

Proof. If $A$ is commutative, then everything in $A^{\cap}$ is simply polar.

For a specific example take $T: A \rightarrow B$ for the embedding

$$
A=\operatorname{comm}_{B}^{2}(a) \subseteq B=B(X)
$$

with $X=\ell_{2}$ and $a \in A$ either the forward or the backward shift, or alternatively the left regular representation $L: A \rightarrow B(A)$. Here of course $\operatorname{comm}^{2}(a)$ is $\operatorname{comm} \operatorname{comm}(K)$ with $K=\{a\}$. Conversely, however, if $A=B(X)$ for a normed space $X$, then there is the implication

$$
L_{a} A=\operatorname{cl} L_{a} A \Longrightarrow a(X)=\operatorname{cl} a(X) .
$$

Indeed if $a x_{n} \rightarrow y$ and $\varphi \in X^{*}$ and $\varphi(x)=1$, then, with $\varphi \odot y: w \mapsto \varphi(w) y$,

$$
L_{a}(\varphi \odot y)=L_{a}(b) \Longrightarrow y=a(b x) .
$$

For another example observe that generalized permanence fails for the process of factoring out the radical of a ring unless it is semisimple:

$$
\operatorname{Rad}(A) \cap A^{\cap}=O .
$$

\section{Moore-Penrose Permanence}

We recall that a "C* algebra" is a Banach algebra which also has an involution $a \mapsto a^{*}$ which is conjugate linear, reverses multiplication, respects the identity and satisfies the "B* condition"

$$
\left\|a^{*} a\right\|=\|a\|^{2} \quad(a \in A) .
$$

Historically the term " $\mathrm{C}^{*}$ algebra" was reserved for closed *-subalgebras of the algebras $B(X)$ for Hilbert spaces $X$; however the Gelfand-Naimark-Segal (GNS) representation

$$
\Gamma: A \rightarrow B\left(\Xi_{A}\right)
$$

takes an arbitrary "B* algebra" $A$ isometrically into the algebra of operators on a rather large Hilbert space $\Xi_{A}$ built from its "states". A defect of (4.2) would be that if $A=B(X)$, already we do not get $\Xi_{A}=X$ back. In the opinion of at least one writer these terms "B* algebra" and "C* algebra" could easily ([4, Chapter 8$])$ have been Hilbert algebra. When in particular $A=B(X)$ for a Hilbert space $X$, then the closed range condition is sufficient for relative regularity $a \in A^{\cap}$. Indeed we can satisfy (2.2) by setting

$$
b(x)=b(q x), \quad b(a x)=p(x)(x \in X),
$$

where $q^{*}=q=q^{2}$ and $p^{*}=p=p^{2}$ are the orthogonal projections on the range $a(X)$ and the orthogonal complement $a^{-1}(0)^{\perp}$ of the null space. The element $b \in A$ given by (4.3) satisfies the four conditions

$$
a=a b a, \quad b=b a b, \quad(b a)^{*}=b a, \quad(a b)^{*}=a b
$$


and is known as the Moore-Penrose inverse of $a \in B(X)$. More generally in a $\mathrm{C}^{*}$ algebra $A$ the conditions (4.4) uniquely determine at most one element

$$
b=a^{\dagger} \in A,
$$

lying ([5, Theorem 5]) in the double commutant of $\left\{a, a^{*}\right\}$, and are still known as a "Moore-Penrose inverse" for $a \in A$. Now it is a result of Harte and Mbekhta ([5, Theorem 6]) that generally there is equality, writing $A^{\dagger}$ for the set of all MoorePenrose invertible elements of $A$,

$$
A^{\cap}=A^{\dagger}
$$

In an arbitrary $\mathrm{C}^{*}$ algebra, every relatively regular element has a Moore-Penrose inverse. The argument and a slight generalization proceed with the aid of the Drazin inverse. Generally for a $\mathrm{C}^{*}$ algebra $A$ we write

$$
\operatorname{Re}(A)=\left\{a \in A: a^{*}=a\right\}
$$

for the subspace of hermitian elements. Now we claim the following.

Theorem 4.1. If $A$ is a $C^{*}$ algebra, then

$$
\operatorname{Re}(A) \cap A^{\cap} \subseteq \mathrm{QP}(A)
$$

and

$$
a \in A^{\cap} \Longrightarrow a^{*} a \in A^{\cap} \cap \mathrm{QP}(A) .
$$

Proof. When $A=B(X)$, the "ascent" conditions (2.10) hold for hermitian elements $a \in \operatorname{Re}(A)$. This is because [6] generally, if $a \in B(X)$,

$$
x \in X \Longrightarrow\|a x\|^{2} \leq\|x\|\left\|a^{*} a x\right\|,
$$

giving $a^{-1}(0)=\left(a^{*} a\right)^{-1}(0)$. Also if $a=a^{*}$ has closed range, then also the "descent" conditions (2.11) hold, i.e.,

$$
\operatorname{cl} a(X)=\left(a^{*}\right)^{-1}(0)^{\perp} \Longrightarrow \operatorname{cl} a(X)+\left(a^{*}\right)^{-1}(0)=X,
$$

giving also that if $a$ has closed range, $a^{*}(X)=a^{*} a(X)$, which is therefore also closed. To establish (4.8) and (4.9) for general $A$, look at isometric $T: A \rightarrow B(X)$, recalling the boundary condition (1.12), hence spectral permanence and then Drazin permanence.

Theorem 4.1] says that selfadjoint $\mathrm{C}^{*}$ elements have [2] "property EP".

Our main result is a slight generalization and a new proof of the Harte-Mbekhta result (4.6), and at the same time "generalized permanence", equality in (2.4), for isometric $\mathrm{C}^{*}$ homomorphisms:

Theorem 4.2. If $T: A \rightarrow B$ is an isometric $C^{*}$ homomorphism, then it has both the "left" and the "right" Gelfand property, and hence also Drazin permanence; there is equality

$$
A^{\dagger}=T^{-1} B^{\cap} .
$$

Proof. If $a \in A$, then the product $a^{*} a \in A^{+} \subseteq \operatorname{Re}(A)$ is positive, with

$$
\partial \sigma_{A}\left(a^{*} a\right)=\sigma_{A}\left(a^{*} a\right) \subseteq[0, \infty) \subseteq \mathbf{R},
$$

giving "spectral permanence at" $a^{*} a$. Since also

$$
a \in A_{\text {left }}^{-1} \Longleftrightarrow a^{*} a \in A^{-1}
$$

this converts to equality in both the left and the right version of (1.10). 
Now by (4.9) the product $a^{*} a \in \mathrm{QP}(A)$ has a Koliha-Drazin inverse, and even a group inverse, but now (cf. [5, Theorem 10])

$$
a^{\dagger}=\left(a^{*} a\right)^{\times} a^{*}=\left(a^{*} a\right)^{\dagger} a^{*}
$$

gives a Moore-Penrose inverse $a^{\dagger}$ for $a \in A$.

In the situation of (4.8) we now have more detail:

$$
a=a^{*} \in A^{\cap} \Longrightarrow a^{\dagger}=a^{\times}, \quad 1-a^{\dagger} a=a^{\bullet} .
$$

We remark that it is rather easy ([5, Theorem 7]) to see

$$
a \in A^{\dagger} \Longrightarrow a^{*} a \in A^{\cap} \text {, }
$$

for if $b=a^{\dagger}$, then

$$
a^{*} a=a^{*} b^{*} a^{*} a b a=a^{*}(a b)^{*}(a b)^{*} a=a^{*} a\left(b b^{*}\right) a^{*} a .
$$

Theorem 4.2 has an obvious extension to homomorphisms with closed range:

Theorem 4.3. If $T: A \rightarrow B$ has closed range, then there is the implication, for arbitrary $a \in A$,

$$
T(a) \in B^{\cap} \Longrightarrow a+T^{-1}(0) \in\left(A / T^{-1}(0)\right)^{\cap} .
$$

Proof. Apply Theorem 4.2 to $T^{\wedge}: A / T^{-1}(0) \rightarrow B$, which is bounded below.

\section{IrReducible REPRESEntations}

If $T: A \rightarrow B(X)$ is an isometric * homomorphism with a Hilbert space $X$, and if there is a closed subspace $Y \subseteq X$ for which, for arbitrary $a \in A$, there is the inclusion

$$
T(a) Y \subseteq Y, \quad T(a) Y^{\perp} \subseteq Y^{\perp},
$$

then we shall say that the representation has been reduced to a representation $T_{Y}: A \rightarrow B(Y)$. If it follows from (5.1) that

$$
Y=X \text { or } Y=O \equiv\{0\},
$$

then we shall describe the representation $T: A \rightarrow B(X)$ as irreducible. Now if $B(X)$ is the bounded operators on a Banach space, we shall write $B_{00}(X)$ for the ideal of finite rank operators and $B_{0}(X)$ for the larger closed ideal of compact operators. We remark that ([4, Theorem 6.8.5])

$$
B_{0}(X) \cap B(X)^{\cap}=B_{00}(X):
$$

a compact operator is relatively regular if and only if it is of finite rank. When $X$ is a Hilbert space, then $B_{0}(X)=\mathrm{cl} B_{00}(X)$ is also the norm closure of the finite rank operators, which in turn are the linear subspace generated by the rank one operators:

$$
X^{*} \odot X=\left\{x^{*} \odot y: x, y \in X\right\},
$$

where for arbitrary $x, y \in X$ we set

$$
\left(x^{*} \odot y\right)(w)=\langle w ; x\rangle y \quad(w \in X) .
$$

Irreducible $\mathrm{C}^{*}$ subalgebras $A \subseteq B(X)$ have an important property ([1, Theorem 6.3.3]) relating them to the compact ideal $B_{0}(X)$; there is the implication

$$
A \cap B_{0}(X) \neq O \Longrightarrow B_{0}(X) \subseteq A \text {. }
$$


In words, if $A$ contains even one nonzero compact operator, then it contains them all.

As a companion to (5.6) we observe (cf. [1, Theorem 5.2.1]) that if $J \subseteq A$ is a two-sided ideal, there is the implication, when $A=B(X)$,

$$
J \neq O \Longrightarrow A \cap B_{00}(X) \subseteq J .
$$

In words, a nontrivial two-sided ideal of irreducible $A$ contains all the finite rank operators in $A$. If in particular the ideal is closed, then it contains all the compact operators.

If $T: A \rightarrow B$ is a homomorphism of rings, then it also brings ([4, Chapter 7]) a Fredholm theory to the departure ring $A$; generally for $T: A \rightarrow B$ to have the Gelfand property means that "Fredholm implies invertible". Intermediate between the invertibles and the Fredholms is the semigroup of Weyl elements:

$$
A^{-1} \subseteq A^{-1}+T^{-1}(0) \subseteq T^{-1}\left(B^{-1}\right) \subseteq A .
$$

More generally if we replace invertibles by left and right invertibles, we get "left" and "right" Fredholm and Weyl elements. For example, if, with a Banach space $X$,

$$
T=\pi: B(X) \rightarrow D=B(X) / B_{0}(X)
$$

is the Calkin quotient, then (Atkinson's theorem) the Fredholm operators are those with finite dimensional null space and closed range of finite codimension, while (Schechter's theorem) the Weyl operators are the Fredholm operators "of index zero", for which those two finite dimensions are equal. If instead

$$
T=\pi_{0}: B(X) \rightarrow D_{0}=B(X) / B_{00}(X),
$$

then, since

$$
B_{00}(X) \subseteq B(X)^{\cap}, \quad \pi_{0} B_{0}(X) \subseteq \operatorname{Rad}\left(D_{0}\right),
$$

the Fredholm theory is unchanged; there is, recalling (2.5), equality

$$
\pi^{-1} D^{-1}=\pi_{0}^{-1} D_{0}^{-1} \subseteq B(X)^{\cap} .
$$

Now if $A \subseteq B(X)$ is irreducible and more generally if $J \subseteq A$ is a nontrivial closed two-sided ideal, then the homomorphisms

$$
\pi_{A}: A \rightarrow D=B(X) / B_{0}(X)
$$

and

$$
\rho_{J}: A \rightarrow D_{J}=A /\left(J \cap B_{0}(X)\right)
$$

generate, according to [1, Theorem 6.3.4], the same Fredholm theory:

Theorem 5.1. If $A \subseteq B(X)$ is irreducible and

$$
O \neq A J+J A \subseteq J \neq A,
$$

then there is the equality

$$
\pi_{A}^{-1} D^{-1}=\rho_{J}^{-1} D_{J}^{-1} .
$$

Proof. It is clear at once that if $\rho_{J}(a) \in A /\left(J \cap B_{0}(X)\right)$ is left or right invertible, then so is $\pi_{A}(a) \in D=B(X) / B_{0}(X)$. Conversely if $\pi(a)=\pi_{A}(a) \in D_{\text {left }}^{-1}$, then also $\pi_{0}(a) \in\left(D_{0}\right)_{\text {left }}^{-1}$, giving $a \in A^{\cap}$, hence from (4.9) $a^{*} a \in \operatorname{QP}(A) \cap A^{\cap}$, with spectral projection

$$
p=\left(a^{*} a\right)^{\bullet} \in J \cap B_{00}(X),
$$


appealing to irreducibility and (5.7), with

$$
1=\left(\left(a^{*} a+p\right)^{-1} a^{*}\right) a+\left(a^{*} a+p\right)^{-1} p \in A a+J \cap B_{00}(X) .
$$

It follows that $\rho_{J}(a) \in\left(D_{J}\right)_{\text {left }}^{-1}$.

Similarly $\pi_{A}(a) \in D_{\text {right }}^{-1}$ gives $\rho_{J}(a) \in\left(D_{J}\right)_{\text {right }}^{-1}$.

Alternatively, if $\pi_{A}(a) \in D_{\text {left }}^{-1}$, then $a \in A$ has a Moore-Penrose inverse $a^{\dagger} \in A$, and now

$$
1-a^{\dagger} a \in A \cap B_{00}(X) \subseteq J
$$

is the orthogonal projection with finite dimensional range $a^{-1}(0)$.

We remark that Theorem 5.2.1 of [1] holds for $A=B(X)$, while if (5.7) holds and there are any nonzero finite rank operators in $A$, they will all be in $J$. It seems to us that the argument of [1] requires our (5.7) rather than Theorem 5.2.1. of [1]. One might hope to derive our (5.7) by a combination of the arguments of (5.2.1) and (6.3.3), both of [1].

\section{REFERENCES}

1. S. R. Caradus, W. E. Pfaffenberger and B. Yood, Calkin algebras and algebras of operators on Banach spaces, Dekker, 1974. MR0415345 (54:3434)

2. D. S. Djordjević, J. J. Koliha and I. Straskraba, Factorization of EP elements of $C^{*}$-algebras, Linear Multilinear Algebra 57 (2009), 587-594. MR2543720 (2010m:46085)

3. D. S. Djordjević and V. Rakočević, Lectures on generalized inverses, University of Niš, 2008. MR2472376 (2009h:47002)

4. R. E. Harte, Invertibility and singularity, Dekker, 1988. MR0920812 (89d:47001)

5. R. E. Harte and M. Mbekhta, On generalized inverses in $C^{*}$-algebras, Studia Math. 103 (1992), 71-77. MR 1184103 (93i:46097)

6. R. E. Harte and M. O'Searcoid, Positive elements and the $B^{*}$ condition, Math. Zeit. 193 (1986), 1-9. MR852905 (87k:46111)

7. J. J. Koliha, A generalized Drazin inverse, Glasgow Math. Jour. 38 (1996), 367-381. MR 1417366 (98b:46065)

8. X. Mary, On the converse of a theorem of R. Harte and M. Mbekhta, Studia Math. 184 (2008), 149-151. MR2365807 (2008k:46167)

9. M. Takesaki, Theory of operator algebras. I, Springer, 2002. MR.1873025 (2002m:46083)

Department of Mathematics and Informatics, Faculty of Sciences and Mathematics, University of Niš, Niš 18000 , SERBIA

E-mail address: dragan@pmf.ni.ac.rs; dragandjordjevic70@gmail.com

Department of Mathematics and Informatics, Faculty of Sciences and Mathematics, UNIVERSITY OF Niš, Niš 18000 , SERBIA

E-mail address: mladvlad@open.telekom.rs

School of Mathematics, Trinity College, Dublin 2, Ireland

E-mail address: rharte@maths.tcd.ie 